\title{
Cluster observations of high-frequency waves in the exterior cusp
}

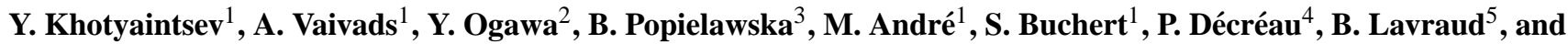 \\ H. Rème ${ }^{5}$ \\ ${ }^{1}$ Swedish Institute of Space Physics, Uppsala, Sweden \\ ${ }^{2}$ Nagoya University, Japan \\ ${ }^{3}$ Space Research Center, Warsaw, Poland \\ ${ }^{4}$ LPCE/CNRS, Orleans, France \\ ${ }^{5} \mathrm{CESR}$, Toulouse, France
}

Received: 8 October 2003 - Revised: 26 May 2004 - Accepted: 24 June 2004 - Published: 14 July 2004

Part of Special Issue "Spatio-temporal analysis and multipoint measurements in space"

\begin{abstract}
We study wave emissions, in the frequency range from above the lower hybrid frequency up to the plasma frequency, observed during one of the Cluster crossings of a high-beta exterior cusp region on 4 March 2003. Waves are localized near narrow current sheets with a thickness a few times the ion inertial length; currents are strong, of the order of $0.1-0.5 \mu \mathrm{A} / \mathrm{m}^{2}\left(0.1-0.5 \mathrm{~mA} / \mathrm{m}^{2}\right.$ when mapped to ionosphere). The high frequency part of the waves, frequencies above the electron-cyclotron frequency, is analyzed in more detail. These high frequency waves can be broad-band, can have spectral peaks at the plasma frequency or spectral peaks at frequencies below the plasma frequency. The strongest wave emissions usually have a spectral peak near the plasma frequency. The wave emission intensity and spectral character change on a very short time scale, of the order of $1 \mathrm{~s}$. The wave emissions with strong spectral peaks near the plasma frequency are usually seen on the edges of the narrow current sheets. The most probable generation mechanism of high frequency waves are electron beams via bump-on-tail or electron two-stream instability. Buneman and ion-acoustic instability can be excluded as a possible generation mechanism of waves. We suggest that high frequency waves are generated by electron beams propagating along the separatrices of the reconnection region.
\end{abstract}

Key words. Magnetospheric physics (magnetopause, cusp and boundary layers) - Space plasma physics (wave-particle interactions; magnetic reconnection)

\section{Introduction}

Magnetospheric cusps are the primary region of magnetosheath plasma entry; they are very dynamic regions with a variable plasma structure (Haerendel and Paschmann, 1975). Cusps are also associated with strong plasma wave activity ranging from very low frequencies (Savin et al., 2004) up to electron-cyclotron and plasma frequencies (Pickett et al., 2001). In most cases the source of the waves and their plasma wave modes are still not known.

One can identify several magnetospheric regions when satellites are crossing the high altitude cusp. One region that stands out is the exterior cusp. This region has been the subject of various terminology (Haerendel, 1978; Savin et al., 2004; Lavraud et al., 2002). Here we define it as the low magnetic field region located at large radial distances. It has recently been shown as more or less stagnant under northward IMF (Lavraud et al., 2002) while rather largely convective under southward IMF (Cargill et al., 2004). It often shows, however, disordered plasma flows and fields (Haerendel, 1978; Savin et al., 2004) and transient flows associated with recently reconnected flux tubes (Khotyaintsev et al., 2004).

We study waves inside the high altitude cusp in a wide frequency range from above the lower hybrid frequency up to the plasma frequency. Bursty whistler emissions with frequencies between lower hybrid and electron-cyclotron frequencies have been reported earlier, for example, from Polar spacecraft (Pickett et al., 2001). Another kind of high frequency waves that are often present have frequencies from the electron-cyclotron up to the plasma frequency. These waves can have spectral peaks near the plasma frequency or have broad-band character. In most cases electron beams have been suggested as the source of the waves (Pickett et al., 2001). There are many studies theoretically and numerically investigating possible ways to generate the waves described above by drifting electrons or electron beams (Roth and Hudson, 1986; Watanabe and Oya, 1993). At the same time it is necessary to have more detailed observations of the waves and their plasma environment in order to answer the questions why the waves are so bursty, what is the temporal and spatial development of the wave intensities and spectra, what are the sources of the electron beams. This paper is a step in this direction. 

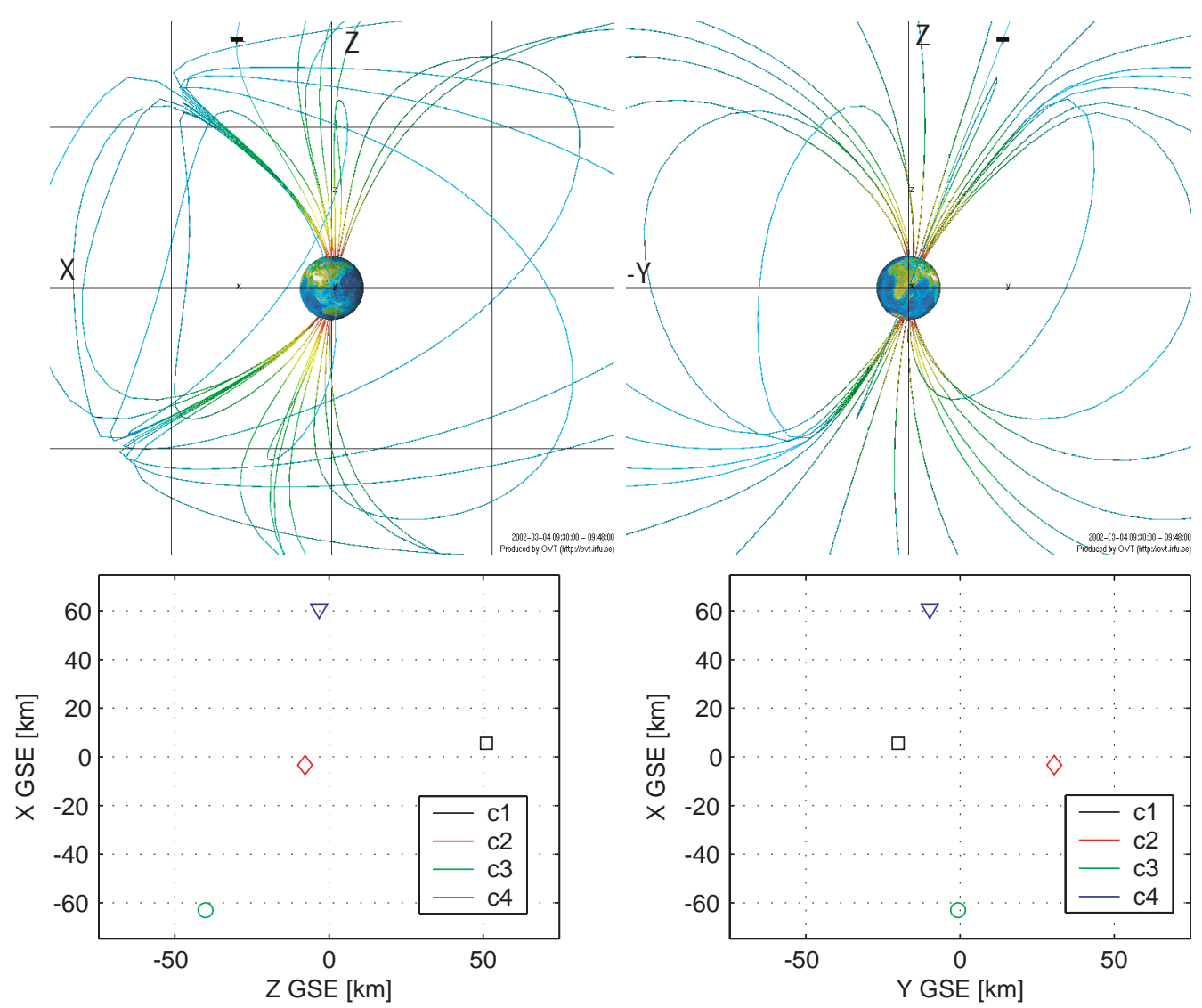

Fig. 1. Location of Cluster spacecraft (marked by a black bar) in the magnetosphere at 09:35 UT: X-Z (right) and Y-Z (left) views and Cluster configuration in GSE (lower panels). The figure is produced with Orbit Visualization Tool (http://ovt.irfu.se).

A reconnection region generally can be rather turbulent and observations of electromagnetic waves in the vicinity of it can provide important information about the physics of the reconnection processes. Instabilities driving waves with frequencies near the electron plasma frequency, such as bumpon-tail and Buneman instabilities, provide information about the electron dissipation. Electron holes, Langmuir and upper hybrid waves close to the reconnection X-line and separatrices are reported from simulations (Drake et al., 2003), satellite observations at the magnetopause (Matsumoto et al., 2003; Vaivads et al., 2004) and the magnetotail (Farrell et al., 2002). Electrostatic instabilities, such as lower hybrid drift instability (LHDI, Krall and Liewer (1971); Silveira et al. (2002)), are commonly observed in association with reconnection current sheets. LHDI is observed in low- $\beta$ regions of the current sheet, but not in the center, where $\beta$ is high and the instability is suppressed (Horiuchi and Sato, 1999; Rogers et al., 2000; Carter et al., 2002). In a high- $\beta$ plasma electromagnetic instabilities play the most important role. One such instability is a modified two-stream instability (MTSI) (Krall and Liewer, 1971; Wu et al., 1983; Silveira et al., 2002), which results from a relative drift of magnetized electrons and unmagnetized ions $\left(v_{d} \sim V_{A}\right)$. Recently, Ji et al. (2004) reported on the correlation of whistler waves driven by MTSI and the enhancement of reconnection rates in a high- $\beta$ laboratory plasma. Observation of enhanced whistler- mode waves in association with a reconnection event on the dayside magnetopause was reported by Deng and Matsumoto (2001).

In this paper we present a multipoint observation of wave emissions in the high altitude cusp region, where wave frequencies are between the lower hybrid and electron plasma frequencies. We analyze the spectral character of these waves, the location of the most intense wave emissions with respect to narrow current sheets, and discuss how details of the observations are consistent with the reconnection hypothesis.

\section{Observations}

We are studying a high altitude cusp observed by Cluster at the dusk flank on 4 March 2002. The location of Cluster spacecraft, their configuration and the model magnetospheric field (T2001 model Tsyganenko (2000)) are shown in Fig. 1. Cluster enters the high latitude cusp at 08:50 UT and exits to the magnetosheath at 09:50 UT. The IMF, as observed by ACE, was primarily duskward $\left(B_{y} \sim 12 \mathrm{nT}\right)$, and generally northward $(5 \mathrm{nT})$ with a small southward excursion after 09:40 UT. Our primary interest is a time interval 09:39:0009:40:30 UT when several of the most intense high frequency wave emissions during the whole cusp passage are observed. 


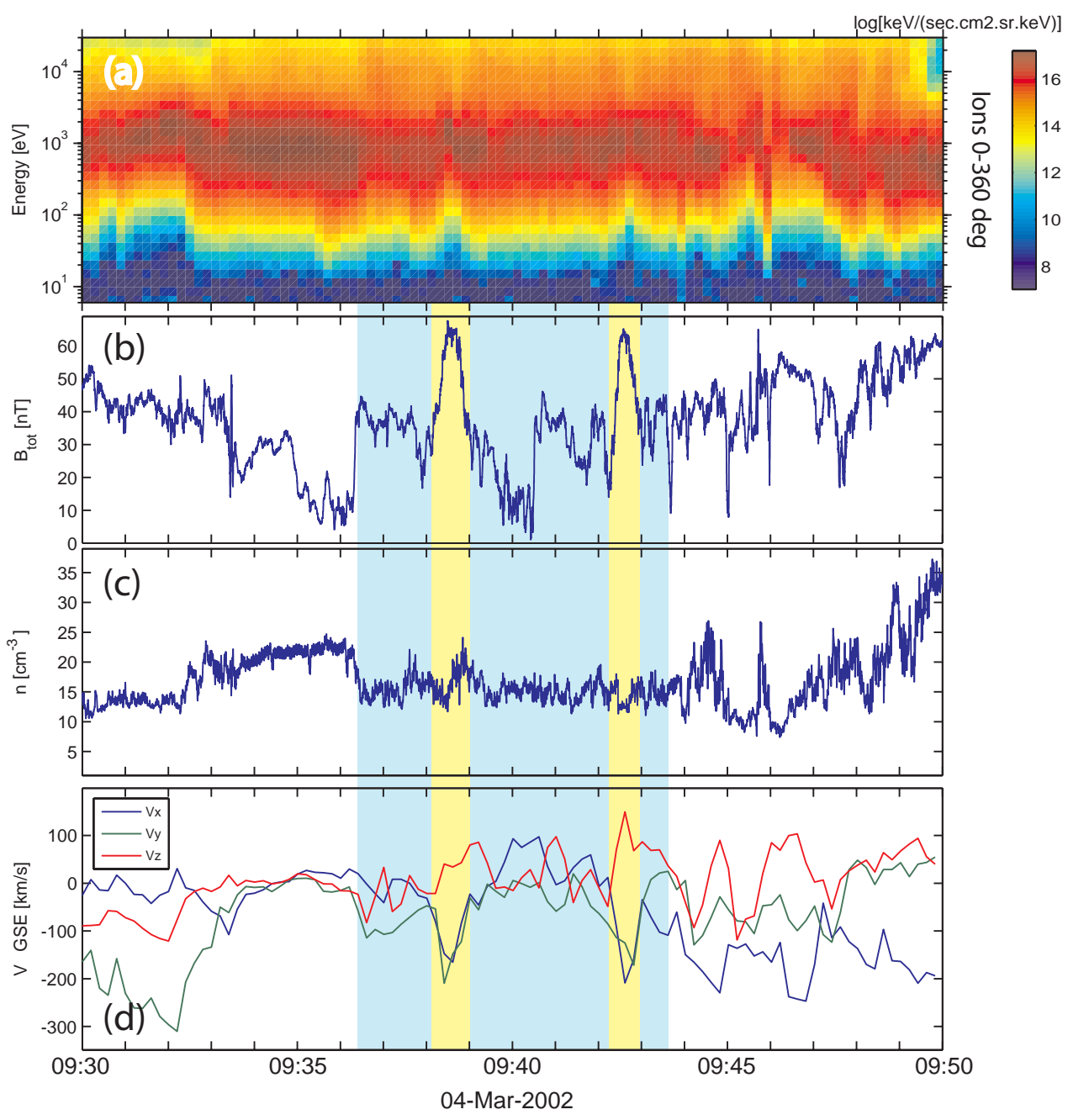

Fig. 2. Overview of the cusp/exterior cusp region observed by Cluster 3 during period 09:30-09:50 UT. Panels from top to bottom show: (a) ion spectrogram, (b) the magnetic field magnitude, (c) plasma density derived from the spacecraft potential, (d) ion flow velocity. The blue shaded region marks the time interval presented in Fig. 3. The yellow bars mark the FTEs.

An overview of an outbound crossing of the cusp region by Cluster 3 is shown in Fig. 2. Panels from top to bottom show: (a) ion spectrogram for all pitch angles measured by HIA (Rème et al., 1997), (b) the magnetic field magnitude measured by FGM (Balogh et al., 1997), (c) plasma density derived from the spacecraft potential measured by EFW (Gustafsson et al., 1997), (d) ion flow velocity measured by HIA (Rème et al., 1997). The exit to the magnetosheath can be identified at $\sim 09: 49$ UT, when the plasma flow becomes tailward (-X GSE) with a positive $\mathrm{Y}$ component (which is expected on the dusk flank). The region preceding the magnetosheath can be, for the most part, identified as exterior cusp.

The most varying quantity in Fig. 2 is the magnitude of the magnetic field $\boldsymbol{B}$. We can identify three characteristic levels of $|\boldsymbol{B}|$ : magnetospheric level where $|\boldsymbol{B}| \sim 35-40 \mathrm{nT}$, and levels where $|\boldsymbol{B}|$ is below and above the magnetospheric level. The regions where $|\boldsymbol{B}| \sim 35-40 \mathrm{nT}$ have a magnetic field direction close to the magnetic field expected for the magnetosphere $\left(B_{y}<0, B_{z}<0\right)$. The regions of low magnetic field can be identified as exterior cusp. They are separated from magnetospheric regions by the magnetopause (current sheet). The exterior cusp is filled with high- $\beta$ plasma which is primarily stagnant or flowing sunward. The regions where the magnetic field magnitude is above the magnetospheric level, $|\boldsymbol{B}|>40 \mathrm{nT}$, contain plasma which flows tailward but at the same time has significant velocity component in -Y GSE direction, which is in opposite direction with respect to the IMF $B_{y}$ component. This direction is expected for the flux tubes that are being dragged around the magnetosphere after reconnection. These regions can be interpreted as encounters of transient flows associated with recently reconnected flux tubes (flux transfer events, FTEs, Paschmann et al. (1982)), produced by transient magnetic reconnection on the dusk flank, where the magnetosheath field (having large positive $B_{y}$ ) is close to being antiparallel to the magnetospheric field. Two examples of the FTEs be seen at $\sim$ 09:38:30 and $\sim$ 09:42:30 UT (marked by yellow 

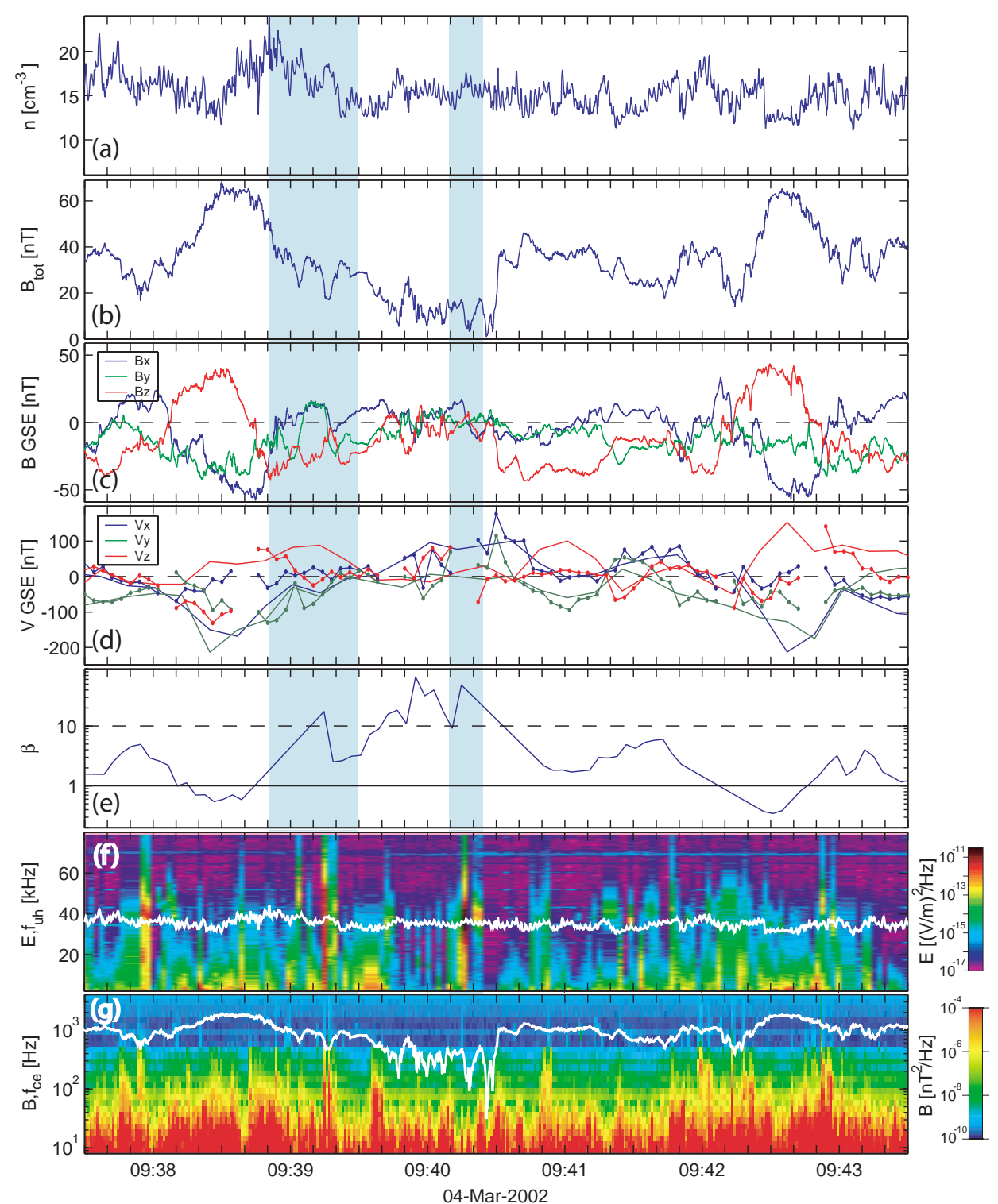

Fig. 3. Detailed view of the cusp/exterior cusp region observed by Cluster 3 during period 09:38-09:41 UT. Panels from top to bottom show: (a) plasma density derived from the spacecraft potential, (b, c) the magnetic field magnitude and components, (d) ion flow velocity (full from HIA, solid line, and $\boldsymbol{E} \times \boldsymbol{B}$, dotted line), (e) plasma $\beta$, (f) spectra of the electric field in $2-80 \mathrm{kHz}$ range and the upper hybrid frequency, (g) spectra of the magnetic field in $8-4000 \mathrm{~Hz}$ range and the electron-cyclotron frequency. The shaded regions mark the time intervals presented in Fig. 4.

bars in Fig. 2). The high magnetic field amplitudes inside the FTEs are similar to the magnetic field amplitude observed in the magnetosheath; see Fig. 2 after 09:49 UT. However, the direction of the magnetic field inside the FTEs (large negative $B_{y}$ component) is different from the one in the magnetosheath (where one expects positive $B_{y}$ ), and thus, the regions which we define as FTEs cannot be simply interpreted as encounters of the magnetosheath. The FTEs are filled with magnetosheath-like plasma and have a flow direction driven by IMF $B_{y}$ (perpendicular flow in -Y GSE) and the magnetosheath flow (parallel flow in $-X$ GSE). The FTEs are separated by a region of high- $\beta$ plasma of the exterior cusp which is flowing sunward.
We look in more detail at the time interval 09:3809:41 UT, the shaded region in Fig. 2. This time interval contains regions with all three different levels of $\boldsymbol{B}$; the overview of this time interval is shown in Fig. 3. The reason why we have chosen this time interval is that within it we observe among the strongest high frequency waves emissions during the whole high altitude cusp passage. Panels from top to bottom show: (a) plasma density derived from the spacecraft potential measured by EFW, $(b, c)$ the magnetic field magnitude and components measured by FGM, (d) ion flow velocity (HIA, solid line) and $\boldsymbol{E} \times \boldsymbol{B}$ from EFW (dotted line), (e) plasma $\beta$ (ion $\beta$ ), (f) spectra of the electric field in $2-80 \mathrm{kHz}$ range measured by WHISPER instrument 

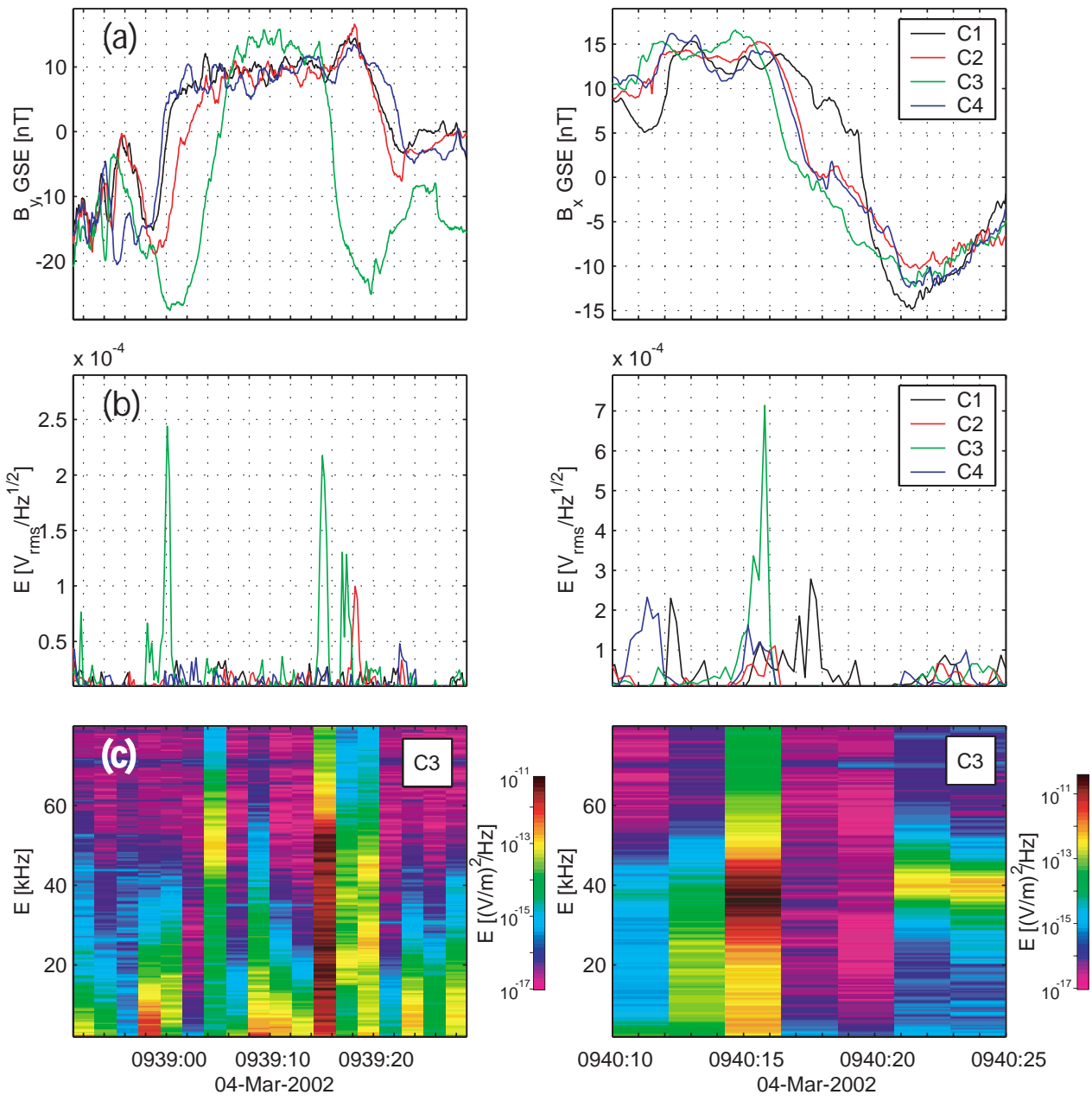

Fig. 4. Two events of enhanced high-frequency waves observed in association with strong localized current sheets. Panels show (a) largest varying component of the magnetic field, (b) integrated spectra of the electric field in a range $2-80 \mathrm{kHz}$ observed by all Cluster spacecraft and (c) spectra of the electric field observed by Cluster 3.

(Decreau et al., 1997) co-plotted with the upper hybrid frequency, $(\mathrm{g})$ spectra of the magnetic field in $8-4000 \mathrm{~Hz}$ range measured by STAFF-SA (Cornilleau-Wehrlin et al., 1997) co-plotted with the electron-cyclotron frequency. We note that during this event the upper hybrid frequency $f_{u h}$ is nearly identical to the electron plasma frequency $f_{p e}$ because of the low electron-cyclotron frequency $f_{c e} \sim 0.2-1 \mathrm{kHz}$.

We would like to concentrate on bursts of highfrequency waves close to the plasma frequency seen on in Fig. 3f. We see several such bursts at 09:37:55, 09:39:05-09:39:15, 09:39:40-09:39:45, 09:40:15-09:39:25 and 09:42:50-09:39:43:00 UT. Some of these bursts (09:37:55, 0939:05-09:39:15, 09:42:50-09:39:43:00 UT) are located at the edges of the FTEs, with boundaries separating regions of high magnetic field $|\boldsymbol{B}|>40 \mathrm{nT}$ and significant plasma flow. Other bursts of high-frequency waves (09:39:40-09:39:45 and 0940:15-09:39:25 UT) are located at the boundaries of high- $\beta$ exterior cups region.

Figure $3 \mathrm{~g}$ shows electromagnetic activity as measured by the STAFF instrument in a frequency range up to $4 \mathrm{kHz}$, which is several times the electron-cyclotron frequency. Bursty emissions with spectral peaks at frequencies below the electron-cyclotron frequency are seen throughout the cusp. These emissions can be identified as whistlers because of their right-hand polarization (not shown). Some of the whistler emissions (09:37:55, 09:39:15, 09:40:22, 09:42:55 UT) are correlated with the high frequency wave emissions.

Figure 4 shows two of the strongest events of waves at frequencies up to the plasma frequency seen by WHISPER at 09:39:15 and 09:40:15 UT (shaded regions in Fig. 3). Both events are related to narrow current sheets with a thickness of about one ion inertial length $\lambda_{i}=c / \omega_{p i}$, and have correlated enhancements of intensity of whistler waves (Fig. $3 \mathrm{~g}$ ). The geometry of the current sheets is complicated and 3dimensional; there are significant differences between the spacecraft even on such a small separation as $100 \mathrm{~km}$. We cannot easily apply methods which assume a planar current sheet, such as minimum variance and timing, to characterize these current sheets. 
The first event (09:38:50-09:39:30 UT) is related to the boundary of the first FTE. Surrounding plasma has $\beta \sim 3$ and a decaying flow in the - Y GSE direction, and the magnitude of the magnetic field is decreasing from $|\boldsymbol{B}| \sim 70 \mathrm{nT}$ down to $\sim 30 \mathrm{nT}$. The spacecraft cross a narrow current sheet several times, seen as in the changes of $B_{y}$ in Fig. 4a. We see large differences in the magnetic field between the spacecraft. This allows us to conclude that the scale of the current sheet is definitely below the spacecraft separation $\left(100 \mathrm{~km}, \lambda_{i} \sim 60 \mathrm{~km}\right)$, as Cluster 3 sees the field direction which is opposite to the other spacecraft. A good minimum variance exists for Cluster 3, which shows the presence of a magnetic field component $(\sim 5 \mathrm{nT})$ in the direction of minimum variance. This may indicate that the observed current sheet is a rotational discontinuity, but this result cannot be fully trusted because of the actual complicated structure of the crossing. The magnetic field remains rather large in the center of the current sheet ( $\sim 20 \mathrm{nT})$, and thus a large fraction of the observed current is field-aligned. The high-frequency waves are concentrated close to the currents, but have a minimum in the center, which is best seen as a gap in the integrated wave energy for Cluster 3 (green line on panel (b), Fig. 4) at 09:39:16 UT. Spectra of the emissions differ significantly, some of which have a single peak at the electron plasma frequency (09:39:04 UT), other are more broad-band (09:39:16 UT).

During the second event (09:40:10-0940:25 UT) all spacecraft cross a localized current sheet at $\sim 09: 40: 18$ UT, seen as a strong change in $B_{x}$ and an associated depression of total B. Cluster 1 observes a significantly different situation from the others. The current sheet is embedded in plasma with $\beta>10$. The magnetic field shear is $\sim 150^{\circ}$ and its magnitude drops significantly in the center of the region; the minimum values go as low as $1 \mathrm{nT}$ (at Cluster 4). In the center of the current sheet where the magnitude of $\boldsymbol{B}$ is very low, plasma $\beta$ is $\sim 70$. The direction of the surrounding plasma flow is primarily sunward without any clear relation to the IMF $B_{y}$ direction. Wave activity in a frequency range from the lower hybrid up to the electron plasma frequency is strongly enhanced in the vicinity of the current sheet.

Understanding the orientation of the current sheet is rather complicated in this case. The largest and smallest variations of the magnetic field are seen in the GSE X and Y components, respectively. This can be verified with minimum variance analysis (MVA), which shows the existence of good quality MV frame for Cluster 2, 3, and 4, with a ratio of intermediate to minimum eigenvalue $>10$. Magnetic fields observed by Cluster 2, 3, and 4 are similar and can be easily shifted in time to overlap. However, the magnetic field seen by Cluster 1 is significantly different and cannot be uniquely aligned with the rest of the spacecraft. One of the possible directions of the normal to the current sheet which can be derived from the measurements on all four spacecraft is obtained by aligning the maximum varying component of the magnetic field ( $B_{x}$, aligning the current sheet in general) and wave energy measured by WHISPER. Such alignment yields a normal velocity of the current sheet of $V_{n}=55-70 \mathrm{~km} / \mathrm{s}$, resulting in a scale of the current sheet of $330-350 \mathrm{~km}$ or $\sim 6 \lambda_{i}$ $\left(\lambda_{i} \sim 60 \mathrm{~km}\right)$.

Main wave activity seen in a frequency range above $2 \mathrm{kHz}$ (panels (b) and (c) in Fig. 4) is concentrated, as for the first event, at the edges of the current sheet. This can be best seen at Cluster 1, which enters the sheet slower than the other spacecraft and stays at the edge for a longer time, and sees the enhanced waves during all this time. We would like to emphasize that these HF waves observed at 09:40:15 UT have the largest amplitude during the whole cusp crossing of $\sim 1 \mathrm{~h}$.

\section{Discussion}

We have presented observations of different kinds of high-frequency waves that are present in the high-latitude cusp/magnetopause region during one event. This magnetospheric region is characterized by a relatively low magnitude of the magnetic field and dense plasma, so that the electron plasma frequency and the upper hybrid frequency are close to each other and are well above the electron-cyclotron frequency $\left(f_{u h} \sim f_{p e} \gg f_{c e}\right)$. The ion plasma frequency is of the order of the electron-cyclotron frequency (a few hundred $\mathrm{Hz}$ ). During the event that we study, there are no time series data of high frequency emissions (events where such information is available is a topic of our future studies), and also, the polarization properties of high frequency waves cannot be estimated. A typical extent of the emissions is $\sim 1 \mathrm{~s}$, which is shorter than the satellite spin period and the temporal resolution of particle distribution functions. Therefore, reliable electron distribution functions from the regions of emissions are not available. Thus, we have to base our analysis and speculations only on wave spectral information.

During this event, the strongest emissions in a frequency range above the electron-cyclotron frequency usually have either a spectral peak near the electron plasma frequency, or a spectral peak at frequencies well below the electron plasma frequency, or broad-band spectra; see examples in Fig. 4c. Different types of wave emissions can be seen just within a few seconds next to each other or even at the same time. Some of these kinds of spectra have been reported earlier, for example, at magnetopause (Matsumoto et al., 2003; Vaivads et al., 2004) and in the magnetotail (Omura et al., 1996; Farrell et al., 2002). In earlier studies the different kind of waves have been identified as electron-acoustic, ion-acoustic, Buneman, Langmuir or upper hybrid waves. It has been identified from the time series measurements that in some cases the broad-band spectra can be due to electron holes (Cattell et al., 2002; Matsumoto et al., 2003).

All of the proposed mechanisms for the generation of high frequency waves gain energy either from the drift of electrons with respect to ions (Buneman, ion-acoustic instabilities) or from the drift of one electron population with respect to another (two-stream, electron-acoustic, weakbeam, bump-on-tail instabilities). Typically under the conditions of dense plasma $\left(f_{p e} \gg f_{c e}\right)$ weak electron beams 
or bump-on-tail distributions would generate upper hybrid waves at oblique propagation angles, and Langmuir waves and electron-acoustic waves propagating parallel to the ambient magnetic field (Roth and Hudson, 1986). For strong electron beams the electron-acoustic waves can start to dominate resulting in spectrum that extends only up to $f_{p e}$ (Gary and Tokar, 1985). Typically, electron-acoustic waves are observed at frequencies that are near the plasma frequency of the cold (non drifting) population of electrons. The electronacoustic waves can evolve into electron holes (Berthomier et al., 2000), leading to broad-band spectra. In the case of a Buneman instability, where electrons move with respect to ions, lower frequency waves of the broad-band character are generated. Electron or ion-holes can also be formed in this case. The missing electron distribution function data does not allow us to observationally identify the free energy source; however, for us it is important that, in principle, all high frequency wave emissions are local electrostatic instabilities that do not propagate far from the generation region; only slight changes in the electron distribution or electron beam strength can create different type of spectra that are seen in our observations.

The high frequency emissions are seen throughout the cusp but the strong emissions are very localized and few. Burst of the wave emissions, which are several second long, are registered approximately once per minute. Most of the strongest emissions that we observe have spectral peaks near the plasma frequency. There are cases when there are no spectral peak near the plasma frequency, but the integrated wave energy suggests strong wave power, e.g. 09:39:00 UT in Fig. 4. In these cases there can be strong waves near the plasma frequency which are not registered, because the spectral information (which is not continuously sampled) happened to be sampled just before or after the region of strong waves. There are also regions of strong broad-band waves that can be seen both in Figs. 3 and 4. Both figures also suggest that broad-band spectra and spectra with peaks near the plasma frequency are often anticorrelated in time, i.e. either the strong Langmuir or strong broad-band emissions are present, but not both at the same time. In our events we cannot distinguish Langmuir and upper-hybrid waves because the polarization information is missing.

The high frequency emissions are observed near strong narrow current sheets; thus, the first thing is to test how important are the current driven instabilities. When we apply the curlometer technique (not shown) we see that currents within the current sheets in Fig. 4 can reach values up to $0.5 \mu \mathrm{A} / \mathrm{m}^{2}$. The component of the current parallel to the magnetic field can be as high as $0.25 \mu \mathrm{A} / \mathrm{m}^{2}$. For typical plasma parameters of density $n=10 \mathrm{~cm}^{-3}$ and current density $j=0.2 \mu \mathrm{A} / \mathrm{m}^{2}$ we obtain an electron drift velocity of $\sim 125 \mathrm{~km} / \mathrm{s}$. This corresponds to a velocity of $80 \mathrm{eV}$ proton which is well below the thermal velocity of protons. The proton temperature is a few hundred of $\mathrm{eV}$ up to $1 \mathrm{keV}$ and thus for the electron drift to be larger than the ion thermal velocity the currents should be at least $\sim 1 \mu \mathrm{A} / \mathrm{m}^{2}$. We cannot exclude the existence of such currents at separations

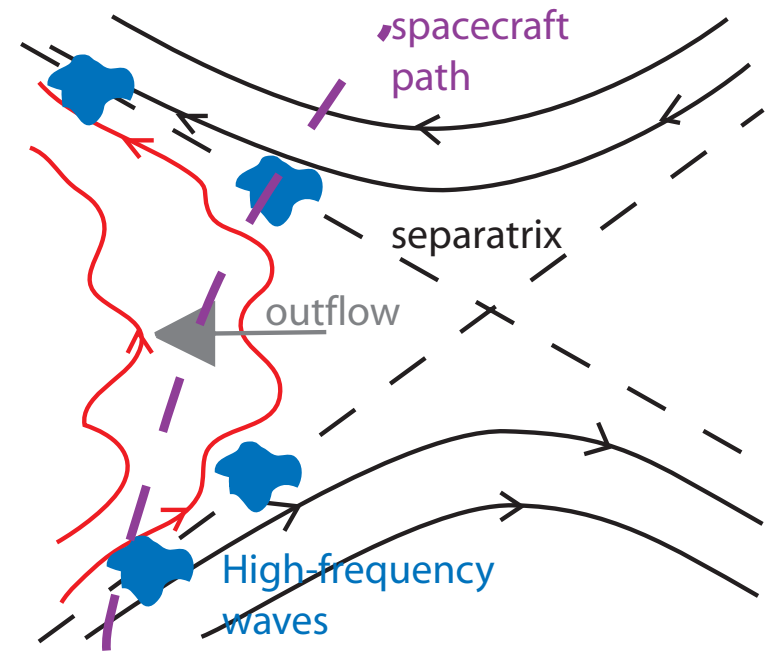

Fig. 5. Schematic of a reconnection region crossing by a spacecraft.

well below the Cluster separation, as the curlometer technique shows only the average current on the Cluster separation scale. While the observed currents of $\sim 0.25 \mu \mathrm{A} / \mathrm{m}^{2}$ are strong currents, and they would correspond to very strong currents of $\sim 0.25 \mathrm{~mA} / \mathrm{m}^{2}$ when mapped to the ionosphere, it does seem that locally these strong currents are not the source of the high frequency emissions. The observations of the high frequency emissions usually at the edges of the current sheets additionally confirm this result. Thus, it seems that high frequency waves are related to the strong narrow current sheets but are not generated by them.

One model that explains reasonably well the above discussed properties of the waves is the presence of reconnection processes close to the satellites. A schematic of the region (near or far away from the $\mathrm{X}$-line) is presented in Fig. 5, where an example satellite trajectory is marked by a dashed line. Electron beams at the separatrices of the reconnection region will generate HF waves which are observed by satellite. Such electron beams streaming toward the X-line along the separatrices have been seen in simulations (Hoshino et al., 2001) and satellite data (Fujimoto et al., 1997; Nagai et al., 2001; Oieroset et al., 2001). We must mention that observation of such electron beams is not possible for the events studied, as the observed structures are rather short and cannot be resolved by particle instruments.

The complicated (turbulent) structure of the observed current sheets does not allow us to show directly whether there is local reconnection taking place. However, some of the current sheets are observed at the edges of the FTEs which also suggests the presence of electron separatrices connected to the $\mathrm{X}$-line.

The lower frequency electromagnetic activity with frequency extending from the lower hybrid frequency $(<10 \mathrm{~Hz})$ up to the electron-cyclotron frequency $(\sim 0.2-1 \mathrm{kHz})$, presented in Fig. 3g, correlates with HF activity. Polarization of these waves is mainly right-handed; thus, the waves can be identified as right-hand polarized whistlers. A similar 
observation of whistler waves in high- $\beta$ cusp was made by Polar spacecraft (Pickett et al., 2001). The source of the waves was related to the outflow region of the reconnection site. Ji et al. (2004) have observed right-hand polarized whistler inside the current sheet, which propagates along the current sheet, and becomes trapped in the perpendicular direction, in a high- $\beta$ laboratory plasma. Generation of such whistler waves in a high- $\beta$ plasma can be attributed to MTSI (modified two stream instability), caused by perpendicular currents. MTSI arises due to relative cross-field drift of unmagnetized ions and magnetized electrons. Ions are unmagnetized on a typical scale of a current sheet $\sim \lambda_{i}<\rho_{i}$, and instability is excited at drift velocities exceeding the local Alfvén velocity. The electron drift velocity of $\sim 125 \mathrm{~km} / \mathrm{s}$ seen in our case is typically larger or comparable to the local Alfvén velocity, and thus MTSI can be excited by the observed currents.

\section{Summary and conclusions}

We have analyzed in detail an event on 4 March 2002, where Cluster spacecraft, being located at close separation $(\sim 100 \mathrm{~km})$, cross the cusp/exterior cusp region. The observations show enhancements of wave activity in a frequency range from the lower hybrid up to the upper hybrid frequencies. The enhancements are localized at narrow current sheets on a scale of several ion inertial lengths. These current sheets are associated with boundaries separating different regions, such as FTEs, magnetosphere and exterior cusp. The intensity of the currents $\sim 0.25 \mu \mathrm{A} / \mathrm{m}^{2}$ is rather high if compared to the typical intensities observed at the magnetopause $\sim 50 \mathrm{nA} / \mathrm{m}^{2}$ (Dunlop et al., 2002), and would correspond to very strong currents of $\sim 0.25 \mathrm{~mA} / \mathrm{m}^{2}$ when mapped to the ionosphere.

Wave emissions in a frequency range extending from the electron-cyclotron frequency up to the plasma frequency can be both broad-band and more localized in frequency. The strongest waves usually have a peak near the plasma frequency. Some of the spectra have a second peak at half of the plasma frequency. The emission intensity and spectral character are bursty; they change on a time scale below 1 sec. We have also observed intensification of electromagnetic waves below the electron-cyclotron frequency, which have some correlation with HF waves, but are observed more frequently. These wave are right-hand polarized whistlers.

The waves in the plasma frequency range can be Langmuir or upper hybrid waves. Spectral peaks at lower frequencies indicate the presence of electron-acoustic waves. Broadband spectra can also be produced by electron-acoustic waves, or coherent structures (electrostatic solitary waves, electron holes). These waves can be generated by electron beam or current instabilities. The resolution of particle instruments does not allow us to resolve this point. Estimates of the electron drift velocities from the current (obtained by curlometer technique) give values lower than the electron and ion thermal velocities. Thus, Buneman and ion-acoustic instabilities can be excluded as a possible generation mech- anism of waves. Therefore, the most probable generation mechanism is electron beams via bump-on-tail or electron two-stream instability.

Strong wave activity close to the plasma frequency are observed at the edges of the current sheets.

We suggest that the observations of high-frequency waves is related to electron separatrices of a reconnection region. In this case one expects strong localized currents, and electron beams along the separatrices located at the edges of these currents. We cannot determine from the data whether the reconnection is going on locally, but see the enhancements of wave activity at the boundaries, which one expects to be related to reconnection, such as edges of FTEs and the cusp/exterior cusp boundary. Our observations of high frequency waves are similar to the observations of upper hybrid waves along the separatrices in the geomagnetic tail (Farrell et al., 2002) and magnetopause (Vaivads et al., 2004).

Acknowledgements. We would like to thank M. Parrot and N. Cornilleau-Wehrlin for providing STAFF data for this study. AV research is supported by Swedish Research Council.

Topical Editor T. Pulkkinen thanks two referees for their help in evaluating this paper.

\section{References}

Balogh, A., Dunlop, M. W., Cowley, S. W. H., Southwood, D. J., Thomlinson, J. G., Glassmeier, K. H., Musmann, G., Lühr, H., Buchert, S., Acuña, M. H., Fairfield, D. H., Slavin, J. A., Riedler, W., Schwingenschuh, K., and Kivelson, M. G.: The Cluster Magnetic Field Investigation, Space Sci. Rev., 79, 65-91, 1997.

Berthomier, M., Pottelette, R., Malingre, M., and Khotyaintsev, Y.: Electron-acoustic solitons in an electron-beam plasma system, Phys. Plasmas, 7, 2987-2994, 2000.

Cargill, P. J., Dunlop, M. W., Lavraud, B., Elphic, R. C., Holland, D. L., Nykyri, K., Balogh, A., Dandouras, I., and Rème, H.: CLUSTER encounters with the high altitude cusp: boundary structure and magnetic field depletions, Ann. Geophys., 22, 1739-1754, 2004.

Carter, T. A., Ji, H., Trintchouk, F., Yamada, M., and Kulsrud, R. M.: Measurement of Lower-Hybrid Drift Turbulence in a Reconnecting Current Sheet, Phys. Rev. Lett., 88, 15 001, 2002.

Cattell, C., Crumley, J., Dombeck, J., Wygant, J. R., and Mozer, F. S.: Polar observations of solitary waves at the Earth's magnetopause, Geophys. Res. Lett., 29, 9-1, 2002.

Cornilleau-Wehrlin, N., Chauveau, P., Louis, S., Meyer, A., Nappa, J. M., Perraut, S., Rezeau, L., Robert, P., Roux, A., de Villedary, C., de Conchy, Y., Friel, L., Harvey, C. C., Hubert, D., Lacombe, C., Manning, R., Wouters, F., Lefeuvre, F., Parrot, M., Pincon, J. L., Poirier, B., Kofman, W., and Louarn, P.: The Cluster Spatio-Temporal Analysis of Field Fluctuations (STAFF) Experiment, Space Sci. Rev., 79, 107-136, 1997.

Decreau, P. M. E., Fergeau, P., Krannosels'kikh, V., Leveque, M., Martin, P., Randriamboarison, O., Sene, F. X., Trotignon, J. G., Canu, P., and Mogensen, P. B.: Whisper, a Resonance Sounder and Wave Analyser: Performances and Perspectives for the Cluster Mission, Space Sci. Rev., 79, 157-193, 1997.

Deng, X. and Matsumoto, H.: Rapid magnetic reconnection in the Earth's magnetosphere mediated by whistler waves, 410, 557560, 2001. 
Drake, J. F., Swisdak, M., Cattell, C., Shay, M. A., Rogers, B. N., and Zeiler, A.: Formation of electron holes and particle energization during magnetic reconnection, Science, 299, 873-877, 2003.

Dunlop, M. W., Balogh, A., Glassmeier, K.-H., and Robert, P.: Four-point Cluster application of magnetic field analysis tools: The Curlometer, J. Geophys. Res., 107, 1384, 2002.

Farrell, W. M., Desch, M. D., Kaiser, M. L., and Goetz, K.: The dominance of electron plasma waves near a reconnection X-line region, Geophys. Res. Lett., 29, 8-1, 2002.

Fujimoto, M., Nakamura, M. S., Shinohara, I., Nagai, T., Mukai, T., Saito, Y., Yamamoto, T., and Kokubun, S.: Observations of earthward streaming electrons at the trailing boundary of a plasmoid, Geophys. Res. Lett., 24, 2893-2896, 1997.

Gary, S. P. and Tokar, R. L.: The electron-acoustic mode, Phys. Fluids, 28, 2439-2441, 1985.

Gustafsson, G., Bostrom, R., Holback, B., Holmgren, G., Lundgren, A., Stasiewicz, K., Ahlen, L., Mozer, F. S., Pankow, D., Harvey, P., Berg, P., Ulrich, R., Pedersen, A., Schmidt, R., Butler, A., Fransen, A. W. C., Klinge, D., Thomsen, M., Falthammar, C.G., Lindqvist, P.-A., Christenson, S., Holtet, J., Lybekk, B., Sten, T. A., Tanskanen, P., Lappalainen, K., and Wygant, J.: The Electric Field and Wave Experiment for the Cluster Mission, Space Sci. Rev., 79, 137-156, 1997.

Haerendel, G.: Microscopic plasma processes related to reconnection, J. Atmos. Terr. Phys., 40, 343-353, 1978

Haerendel, G. and Paschmann, G.: Entry of solar wind plasma into the magnetosphere, in: Physics of the Hot Plasma in the Magnetosphere, edited by Hultqvist, B. and Stenflo, L., Plenum, NY, 23, 1975.

Horiuchi, R. and Sato, T.: Three-dimensional particle simulation of plasma instabilities and collisionless reconnection in a current sheet, Phys. Plasmas, 6, 4565-4574, 1999.

Hoshino, M., Mukai, T., Terasawa, T., and Shinohara, I.: Suprathermal electron acceleration in magnetic reconnection, J. Geophys. Res., 25 979-25 997, 2001.

Ji, H., Terry, S., Yamada, M., Kulsrud, R., Kuritsyn, A., and Ren, Y.: Electromagnetic Fluctuations during Fast Reconnection in a Laboratory Plasma, Phys. Rev. Lett., 92, 115 001, 2004.

Khotyaintsev, Y., Buchert, S., Stasiewicz, K., Vaivads, A., Savin, S., Papitashvili, V. O., Farrugia, C. J., Popielawska, B., and Tung, Y.-K.: Transient reconnection in the cusp during strongly negative IMF $B_{y}$, J. Geophys. Res., A18, A04204, 2004.

Krall, N. and Liewer, P.: Low-frequency instabilities in magnetic pulses, Phys. Rev. A, 4, 2094, 1971.

Lavraud, B., Dunlop, M. W., Phan, T. D., Rème, H., Bosqued, J.-M., Dandouras, I., Sauvaud, J.-A., Lundin, R., Taylor, M. G. G. T., Cargill, P. J., Mazelle, C., Escoubet, C. P., Carlson, C. W., McFadden, J. P., Parks, G. K., Moebius, E., Kistler, L. M., Bavassano-Cattaneo, M.-B., Korth, A., Klecker, B., and Balogh, A.: Cluster observations of the exterior cusp and its surrounding boundaries under northward IMF, Geophys. Res. Lett., 29, 56-1, 2002.

Matsumoto, H., Deng, X. H., Kojima, H., and Anderson, R. R.: Observation of Electrostatic Solitary Waves associated with reconnection on the dayside magnetopause boundary, Geophys. Res. Lett., 30, 59-1, 2003.

Nagai, T., Shinohara, I., Fujimoto, M., Hoshino, M., Saito, Y., Machida, S., and Mukai, T.: Geotail observations of the Hall current system: Evidence of magnetic reconnection in the magnetotail, J. Geophys. Res., 106, 25 929-25 950, 2001.
Oieroset, M., Phan, T., Fujimoto, M., Lin, R., and Lepping, R.: In situ detection of reconnection in the Earth's magnetotail, Nature, 412, 414-417, 2001.

Omura, Y., Matsumoto, H., Miyake, T., and Kojima, H.: Electron beam instabilities as generation mechanism of electrostatic solitary waves in the magnetotail, J. Geophys. Res., 101, 2685-2698, 1996.

Paschmann, G., Haerendel, G., Papamastorakis, I., Sckopke, N., Bame, S. J., Gosling, J. T., and Russell, C. T.: Plasma and magnetic field characteristics of magnetic flux transfer events, J. Geophys. Res., 87, 2159-2168, 1982.

Pickett, J. S., Franz, J. R., Scudder, J. D., Menietti, J. D., Gurnett, D. A., Hospodarsky, G. B., Braunger, R. M., Kintner, P. M., and Kurth, W. S.: Plasma waves observed in the cusp turbulent boundary layer: An analysis of high time resolution wave and particle measurements from the Polar spacecraft, J. Geophys. Res., 106, 19081-19 100, 2001.

Rème, H., Bosqued, J. M., Sauvaud, J. A., Cros, A., Dandouras, J., Aoustin, C., Bouyssou, J., Camus, T., Cuvilo, J., Martz, C., Medale, J. L., Perrier, H., Romefort, D., Rouzaud, J., D‘Uston, C., Mobius, E., Crocker, K., Granoff, M., Kistler, L. M., Popecki, M., Hovestadt, D., Klecker, B., Paschmann, G., Scholer, M., Carlson, C. W., Curtis, D. W., Lin, R. P., McFadden, J. P., Formisano, V., Amata, E., Bavassano-Cattaneo, M. B., Baldetti, P., Belluci, G., Bruno, R., Chionchio, G., di Lellis, A., Shelley, E. G., Ghielmetti, A. G., Lennartsson, W., Korth, A., Rosenbauer, H., Lundin, R., Olsen, S., Parks, G. K., McCarthy, M., and Balsiger, H.: The Cluster Ion Spectrometry (cis) Experiment, Space Sci. Rev., 79, 303, 1997.

Rogers, B. N., Drake, J. F., and Shay, M. A.: The onset of turbulence in collisionless magnetic reconnection, Geophys. Res. Lett., 27, 3157-3160, 2000.

Roth, I. and Hudson, M. K.: Simulations of electron beam excited modes in the high-altitude magnetosphere, J. Geophys. Res., 91, 8001-8010, 1986.

Savin, S., Zelenyi, L., Romanov, S., Sandahl, I., Pickett, J., Amata, E., Avanov, L., Blecki, J., Budnik, E., Büchner, J., Cattell, J. C., Consolini, G., Fedder, J., Fuselier, S., Kawano, H., Klimov, S., Korepanov, V., Lagoutte, D., Marcucci, F., Mogilevsky, M., Nemecek, Z., Nikutowski, B., Nozdrachev, M., Parrot, M., Rauch, J. L., Romanov, V., Romantsova, T., Russell, C. T., Safrankova, J., Sauvaud, J. A., Skalsky, A., Smirnov, V., Stasiewicz, K., Trotignon, J. G., and Yermolaev, Y.: Magnetosheath-cusp interface, Ann. Geophys., 22, 183-212, 2004.

Silveira, O. J., Ziebell, L. F., Gaelzer, R., and Yoon, P. H.: Unified formulation for inhomogeneity-driven instabilities in the lowerhybrid range, Phys. Rev. E, 65, 36 407, 2002.

Tsyganenko, N. A.: Modeling the inner magnetosphere: the asymmetric ring current and region 2 Birkeland currents revisited, J. Geophys. Res., 105, 27 739, 2000.

Vaivads, A., Khotyaintsev, Y., Rogers, B. N., André, M., Décréau, P., Retinó, A., Paschmann, G., Phan, T. D., and Buchert, S. C.: Structure of the magnetic reconnection diffusion region from four-spacecraft observations, Phys. Rev. Lett., in press, 2004.

Watanabe, T. and Oya, H.: Competing processes of electrostatic plasma waves excited by auroral electron beams - Comparison of EXOS D observation results with computer simulations, J. Geophys. Res., 98, $15621,1993$.

Wu, C. S., Winske, D., Papadopoulos, K., Zhou, Y. M., Tsai, S. T., and Guo, S. C.: A kinetic cross-field streaming instability, Phys. Fluids, 26, 1259-1267, 1983. 